\title{
Houston Digestive Health Institute Better Value- Care Today Toward Best One Tomorrow
}

\author{
Atilla Ertan, MD, FACP, AGAF, MACG* \\ Ertan Digestive Disease Center of Excellence, Houston, Texas, USA
}

Submission: March 07, 2017; Published: March 17, 2017

"Corresponding author: Atilla Ertan, MD, FACP, AGAF, MACG, Professor and Interim Chief, Gastroenterology, Hepatology \& Nutrition Division - UT McGovern Medical School, Director, Ertan Digestive Disease Center of Excellence, Houston, Texas, USA, Email: Atilla.Ertan@uth.tmc.edu

\begin{abstract}
In partnership with the UTHealth McGovern Medical School (UTHMMS) and Memorial Hermann-Texas Medical Center (MH-TMC), the Ertan Digestive Disease Center (EDDC) activities continue to be progressively successful, productive and cutting-edge in academic practice, research and postgraduate education since the Center's opening in 2012.

The EDDC delivers high-quality, specialized care to patients from nine different countries, fourteen states, and referrals from all over Texas with the multidisciplinary and integrated approach. Also, this teamwork generates outstanding research and postgraduate education activities.

As part of an academic health center, we have a robust research enterprise aimed at developing the latest treatments and diagnostics for our referral patients. The EDDC and UTHMMS Gastroenterology, Hepatology and Nutrition Division support an active clinical research program and maintains close ties with the Clinical Research Unit of the National Institutes of Health, UTHMMS, MD Anderson Cancer Center (MDACC) for clinical and translational research. The Center's experts have published more than 40-60 articles annually in well-respected medical journals and have presented more than 50-60 studies annually at local and national meetings.

The GI fellowship program is approved by the Accredited Council for Graduate Medical Education. Only 3 to 4 fellows are selected from over 450-500 applicants annually. Our 11 GI fellows rotate to the MH-TMC, the UT MDACC and the Lyndon Baines Johnson General Hospital. These affiliations provide GI fellows with exposure, not only to a large and diverse population, but also to renowned faculty in gastroenterology and liver diseases.
\end{abstract}

\section{Introduction}

One unique and innovative aspect of the multidisciplinary and integrated approach of the EDDC is that it brings together cuttingedge expertise in gastroenterology, hepatology, GI/bariatric and liver-pancreas transplantation surgery, GI pathology, GI radiology, GI oncology and anesthesiology to provide comprehensive academic practice to patients with gastrointestinal and liver diseases. This approach is very complimentary to clinical research and postgraduate education activities.

We are planning on doubling the EDDC in size during 20172018. The entire 14th floor will have routine and therapeutic endoscopy, GI motility and GI physiology labs, and conference room. Another floor, equaling the 23,000 sq.ft. that we currently occupy in the MH Plaza building will be renovated for gastroenterology, hepatology and liver-pancreas transplantation clinics. The success of the EDDC opens an avenue for the desperately needed Houston Digestive Health Institute (HDHI ). The HDHI will be established with excellence in academic practice, research, and postgraduate education with superior quality and high value metrics by both UTHMMS and MH-TMC leaders' rigorous and disciplined work. The UTHMMS selected academicians will provide stellar academic patient value-care, innovative clinical research, and integrated postgraduate education. The TDHI will be complimentary to all involved UTHMMS Departments within available policy, guidelines and academic principles as seen in the few other established US medical schools.

As we all know, there are tremendous changes in the US and in academic medicine. The challenge of becoming a "value based" and dynamic academic medical organization should not be underestimated and this transformation must come from our own leadership. Therefore, the HDHI will have a strategic agenda to achieve the following goals for this transformation;

i. Comprehensive academic practice

ii. Research 


\section{iii. Education and training}

iv. Innovation

v. Quality improvement with high value metrics

The impact of Digestive Health Institutes is very striking at the Virginia Mason Medical Center, the Cleveland Clinic and the Mayo Clinic. We will learn from their experiences and the TDHI agenda will have several components;

\section{Organized integrated centers of excellence}

This requires a cultural shift from today's organization by department to organizing around the patients' medical conditions. These centers of excellence will have both experienced academicians and other supporting personnel to provide for the patients' specific diseases. These teams not only provide diagnosis and treatment, but also assume responsibility for engaging patients and the patients' family in education and counseling for further management, and if needed, provide them with appropriate clinical research protocols. The assigned team's primary goal will be maximizing the patient's overall outcomes as effectively as possible. We will have regular meetings with a team approach to discuss patients, processes, results, management consequences and clinical research outcomes. The HDHI 's combined medical and surgical centers of excellence will be:

i. Esophageal Center of Excellence

ii. Inflammatory Bowel Disease Center of Excellence

iii. Liver Center of Excellence

iv. Pancreas and Biliary Center of Excellence

v. Therapeutic Endoscopy Center of Excellence

vi. Liver and Pancreas Transplantation Center of Excellence

vii. Bariatric Gastroenterology Center of Excellence

viii. Gastroenterology and Hepatology Outcomes Center of Excellence

The care from these centers will be delivered by specialized, dedicated medical and surgical multidisciplinary academicians and teams who devote a significant portion of their efforts to these specific disorders. Efficient, effective and faster treatment with better outcomes, lower costs and high-quality metrics can be achieved through these integrated centers of excellence as observed in EDDC activities within the last 5 years.

\section{Existing and innovative research}

We have a significant number of clinical research programs at the EDDC and some existing basic research development. There is a huge need for standardized data collection. Extension of our existing research program, standardized data collection by development of a common data entry portal, and patient-disease outcome studies will be the Institute's initial focus. Collaboration with UTHMMS basic researchers and additional new basic research
$\mathrm{MD} / \mathrm{PhD}$ faculty will lead to unique combined research protocols. We will initially focus on patients with inflammatory bowel diseases and chronic liver diseases, with plans to expand to further disease processes in the future. This will require us to recruit and support a few funded mid-level and senior-level faculties with NIH grants who are willing to relocate. We also plan to organize a research mentoring program for GI fellows and junior medicine and Surgery faculty at the HDHI . The research portfolio of the TDHI includes pharmaceutical and medical device trials, as well as translational research.

\section{Existing and upgraded postgraduate education}

The infrastructure and culture of the existing successful UTHMMS GI fellowship and advanced endoscopy fellowship programs will be improved with quality, effectiveness and more clinical research protocols. We will establish intermediate and long-term plans on GI postgraduate education and the culture will be rearranged and balanced with a focus on clinical care and more research productivity with a mentorship program. Because the future rests in training and mentoring the next generation of gastroenterologists, the HDHI may add two new fellowships, one in inflammatory bowel disease and the other in therapeutic endoscopy focusing on innovative clinical research activities.

\section{Build an information technology program with quality metrics}

The complexity of the system will require the right kind of IT system that will help with integration, quality communication, and tie the centers with referral physicians as a seamless delivery system. Physicians' notes, lab orders and results, communication documentation, treatment, other data and aspects of care will be stored in a single place. Therefore, everyone participating in the patients' care has access to a real-time comprehensive view and everyone is speaking the same language. The medical records will be accessible to all parties involved in the care of the patient. This IT program makes it easy to extract information including the data needed to measure patient outcomes, not only after their care is completed, but also during care, to enable better clinical decisions and follow the management cost. As provided at the Cleveland Clinic, we will give an opportunity to our patients to have full access to their physicians' notes as another way to improve academic patient care. A proper IT system enables effective collaboration and coordination within the HDHI teams and patients, while also making the extraction, comparison and reporting of outcomes and costs easier.

\section{Arrange the cost of care}

Existing costing systems are fine for overall budgeting, but they provide only crude and quite misleading estimates of actual costs of service for individual patients and conditions. The HDIH academicians do not have enough knowledge of cost of care, much less how costs relate to the outcomes achieved. This cost care arrangement will allow lower costs to attain the same outcomes achieved historically. Cost accounting should be our friend. Understanding true costs will finally allow HDHI academicians to 
work with MH administrators to improve the value of care, which is the fundamental goal of health care organizations.

Health systems in multiple other countries (predominantly in Germany) and a few in the US are moving toward bundled payment approaches. The well- designed bundled payments directly encourage teamwork and high-value care. In the US and also MHTMC, bundled payments have become the norm for organ transplant care. Also, some major employers, such as Walmart, are embracing bundle payments in the US. Moreover, major providers will soon be able to adopt bundles as a tool to grow volume and improve value. The bundle payment method is a huge opportunity for attracting international patients to the HDHI and as is used effectively in few established US academic centers.

\section{Partnership with community gastroenterologists and surgeons}

As seen in major US academic centers, The HDHI will have cooperation and probable partnership with local selected gastroenterologists and surgeons known as Houston GI/Surgery Consortium. This integrated expansion will be focused on improving value as well as increased volume to serve over a wide geographic area.

According our preliminary agreement in the Consortium, local gastroenterologists and surgeons' complex cases will be referred to the HDHI. In addition to complex cases, if complications occur where effective management is beyond the ability of the community gastroenterologists or surgeons, these patients will be transferred to the HDHI. This model is becoming more common among leading academic medical centers and has been very effective at the EDDC within the last five years. Local gastroenterologists and surgeons benefit from the expertise, experience, and reputation of the HDHI academicians. This affiliation will generate numerous opportunities including standardization of the patient management with outlined quality metrics and provide continuous medical education and other benefits to participating local physicians. This approach of the HDHI will be sought out as partners of choice, enabling them to expand across our local regions and beyond with a high-quality value agenda.

\section{Entrepreneurship with our patients and friends}

With shrinking reimbursement, expending regulations in academic practice and research funding, the medical entrepreneurship has an important place and plays an invaluable role for academic progress and improvements. As our recent successful entrepreneurial activities in the EDDC, our interested patients and friends in voluntary basis will be our partners at the HDHI. We will again work with the MH Development Office effective and skillful staff to achieve this goal.

This desperately needed dynamic transformation to a value based modern healthcare delivery system by the HDHI will come from UTHMMS academicians with both UTHMMS and MH-TMC administrators' support to further improve academic value-based practice, clinical research and postgraduate education in Houston and beyond [1-5].

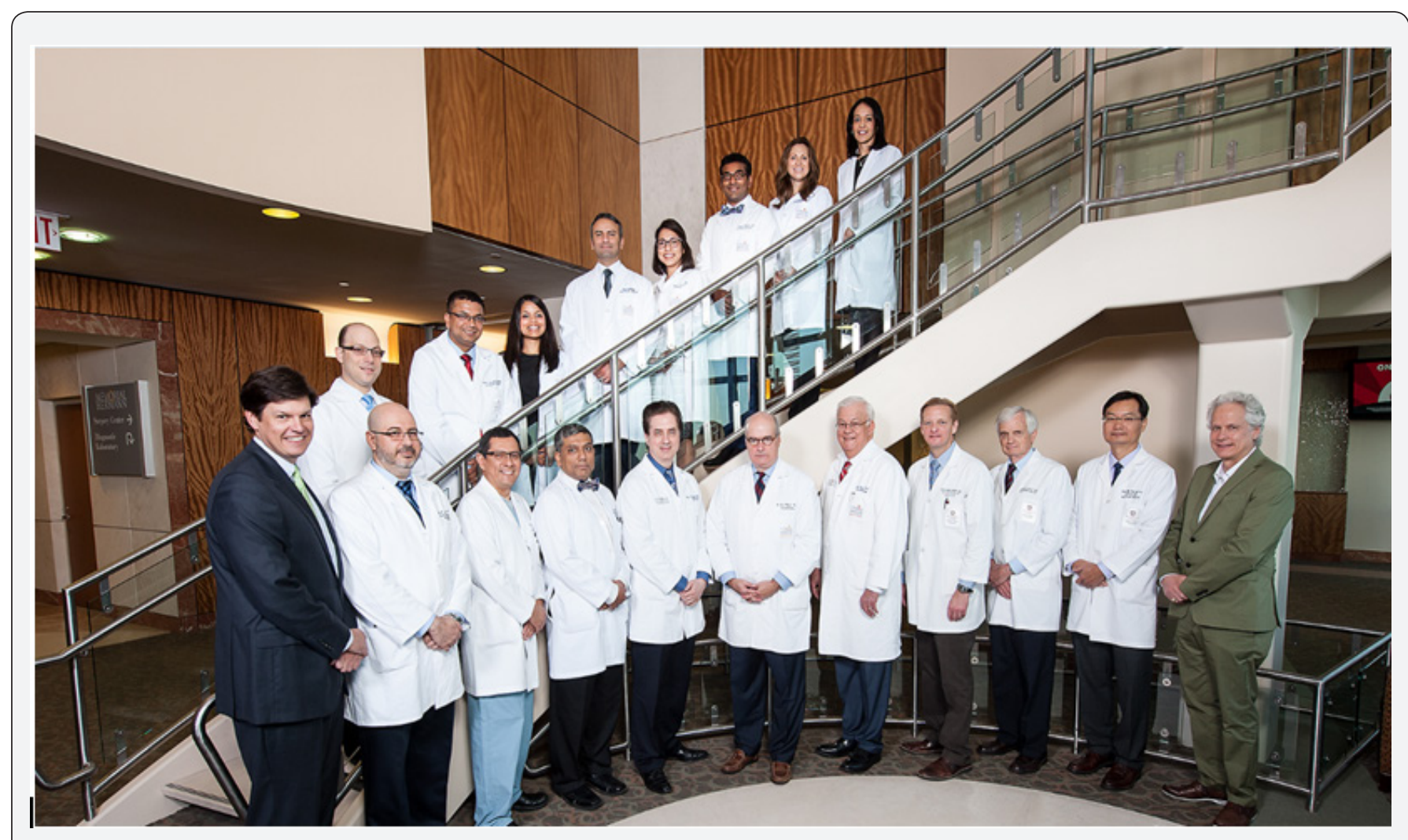




\section{References}

1. Porter ME (2009) A strategy for health care reform-Toward a valuebased system. N Engl J Med 361: 12-15.

2. Porter ME, Lee TH (2013) The strategy that will fix health care. Harvard Business Review 10: 1-58.

3. Rustgi AK, Allen JI (2013) The house of gastrointestinal medicine: how academic medical centers can build a sustainable economic clinic model. Clin Gastroenterol Hepatol 11: 1370-1373.

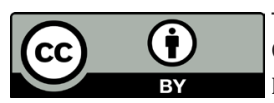

This work is licensed under Creative Commons Attribution 4.0 License DOI: $10.19080 /$ ARGH.2017.04.555627
4. Allen JI, Wang TC, Cohen WS, Shah VH (2016) How to succeed in academic gastroenterology. Gastroenterology 151(4): 578-581.

5. Gardner HK (2017) Getting your stars to collaborate. Harvard Business Review Jan-Feb: 100-108.

\section{Your next submission with Juniper Publishers will reach you the below assets}

- Quality Editorial service

- Swift Peer Review

- Reprints availability

- E-prints Service

- Manuscript Podcast for convenient understanding

- Global attainment for your research

- Manuscript accessibility in different formats

( Pdf, E-pub, Full Text, Audio)

- Unceasing customer service

Track the below URL for one-step submission https://juniperpublishers.com/online-submission.php 